\title{
H-mode characterization for dominant ECRH and comparison to dominant NBI and ICRF heating at ASDEX Upgrade
}

\author{
F. Sommer, J. Stober, C. Angioni, M. Bernert, A. Burckhart, V. Bobkov, \\ R. Fischer, C. Fuchs, R.M. McDermott, W. Suttrop, E. Viezzer \\ and ASDEX Upgrade Team \\ Max-Planck-Institut für Plasmaphysik, EURATOM Association, \\ Boltzmannstraße 2, 85748 Garching, Germany \\ E-mail: fabian.sommer@ipp.mpg.de
}

\begin{abstract}
The influence of pure electron heating versus combined electron and ion heating on a high collisionallity $\mathrm{H}$-mode plasma has been investigated in ASDEX Upgrade. This was done by replacing both neutral beam injection (NBI) and ion cyclotron resonance frequency (ICRF) heating in small steps with electron cyclotron resonance heating (ECRH) while keeping the total heating power constant. The stability of the global plasma parameters and the response of the kinetic profiles on the changed heating mix and torque input is shown. The differences in edge localised mode behaviour between the different heating mixes is reported. The data was analysed with an interpretative transport model and linear gyrokinetic simulations were performed to evaluate the underlying transport mechanism.
\end{abstract}

\section{Introduction}

Present day tokamak plasmas are heated mainly by neutral beam injection (NBI) with beam energies of the order of $100 \mathrm{keV}$ (93 keV and $60 \mathrm{keV}$ at ASDEX Upgrade). In this energy range and with a typical plasma temperature of around 2-3 keV, roughly half of the beam energy is delivered to the electrons and half to the ions. This ratio will change drastically in future plasma experiments like ITER and fusion reactors. To heat the plasma and drive current between the centre and mid radius of the plasma with neutral beams, the beam energy will be increased to much higher values e.g. $1 \mathrm{MeV}$ in ITER. Additionally, the use of electron cyclotron resonance heating (ECRH) will be increased and the heating by $\alpha$-particles (3.5 $\mathrm{MeV}$ ) will start to play a dominant role. All of these changes will shift the balance towards predominantly electron heated plasmas. Since the fusion power and with it the efficiency of a fusion power plant correlates with the triple product $\tau_{E} \cdot T_{i} \cdot n_{D T}$ the aim of any fusion device is to achieve a high ion temperature $T_{i}$ in addition to a high density $n_{D T}$ and energy confinement time $\tau_{E}$. Additionally the relative reduction of the NBI power will lead to a reduced torque input. The resulting lower rotation of the plasma might influence the stability of microturbulence or MHD activity. Also, the deep fuelling by the beams will be negligible in future fusion 
devices. The impact of all these changes on the plasma when switching from NBI to other heating methods is not well understood and therefore, further research is necessary in this area.

The recent upgrade of the ECRH system at ASDEX Upgrade [1], which can now deliver up to $3.9 \mathrm{MW}$ of heating power, provides a practical tool to investigate this matter. Additionally the improved antennas of the ion cyclotron resonance frequency heating (ICRF [2]) system provide greater flexibility in terms of heating scenarios and provide the possibility of deeper insight into this issue. As will be shown in Section 3, the ICRF heating distribution to electrons and ions is very similar to that from the NBI, but its torque input is almost zero, which results in a much smaller rotation of the plasma.

In Section 2 and 3 a comparison of ECRH versus NBI and ECRH versus ICRF heated plasmas is presented. Section 4 describes the initial observations of edge localised mode (ELM [3]) behaviour during the different heating scenarios and Section 5 presents the transport analysis of the discharges. Finally, Section 6 draws some conclusions and gives an outlook on further planned analysis and experiments.

\section{Experimental observations of ECRH versus NBI heated H-modes}

To analyze the influence of different heating schemes on the plasma several discharges were planned and executed in the 2011 campaign at ASDEX Upgrade. An overview of all analysed cases can be seen in Figure 1. All shown cases consist of a phase with at least $500 \mathrm{~ms}$ of constant plasma parameters. Plotted is the heating mix consisting of NBI, ICRF and ECRH against discharge number and time of occurrence in the discharge. The overall range covers a total auxiliary heating power between 2 and $5 \mathrm{MW}$ while the discharges with best comparability are clustered around $3 \mathrm{MW}$. Highlighted are the discharges covered in this paper. \# 27247 comparing ECRH vs. NBI (dashed blue boxes), \# 27246 comparing ECRH vs. ICRH (continuous red boxes), \# 26982 comparing ELM behaviour (dash dotted green boxes) and \# 26457 used for GS2 calculations (dotted purple boxes). In general, heating phases with NBI only lead to a strong Tungsten accumulation, a situation regularly observed at ASDEX Upgrade when no central ECRH is applied [4]. In Figure 2 a strong increase of the concentration of Tungsten $c_{W}$ can be seen at $4.9 \mathrm{~s}$, as soon as the NBI is the only heating and the central ECRH is switched off. For that reason, NBI only phases could only be run at the very end of the discharges, because it leads to modes, unstable discharges and disruptions. The situation for phases with ICRF only heating are worse due to the strong tungsten influx from the W-coated ICRF antennas so that this case could not be analysed. By replacing some of the limiter tiles of the ICRF antennas by Boron coated tiles in the campaign 2012, the Tungsten influx is expected to decrease, so that these experiments can be conducted.

In this section we concentrate on discharge \# 27247 which shows the typical behaviour observed in the experiments. It is a lower single null $\mathrm{H}$-mode with a magnetic field of $B_{t}=-2.5 T$ and a plasma current of $I_{P}=1 M A$, which results in a safety factor at the edge 


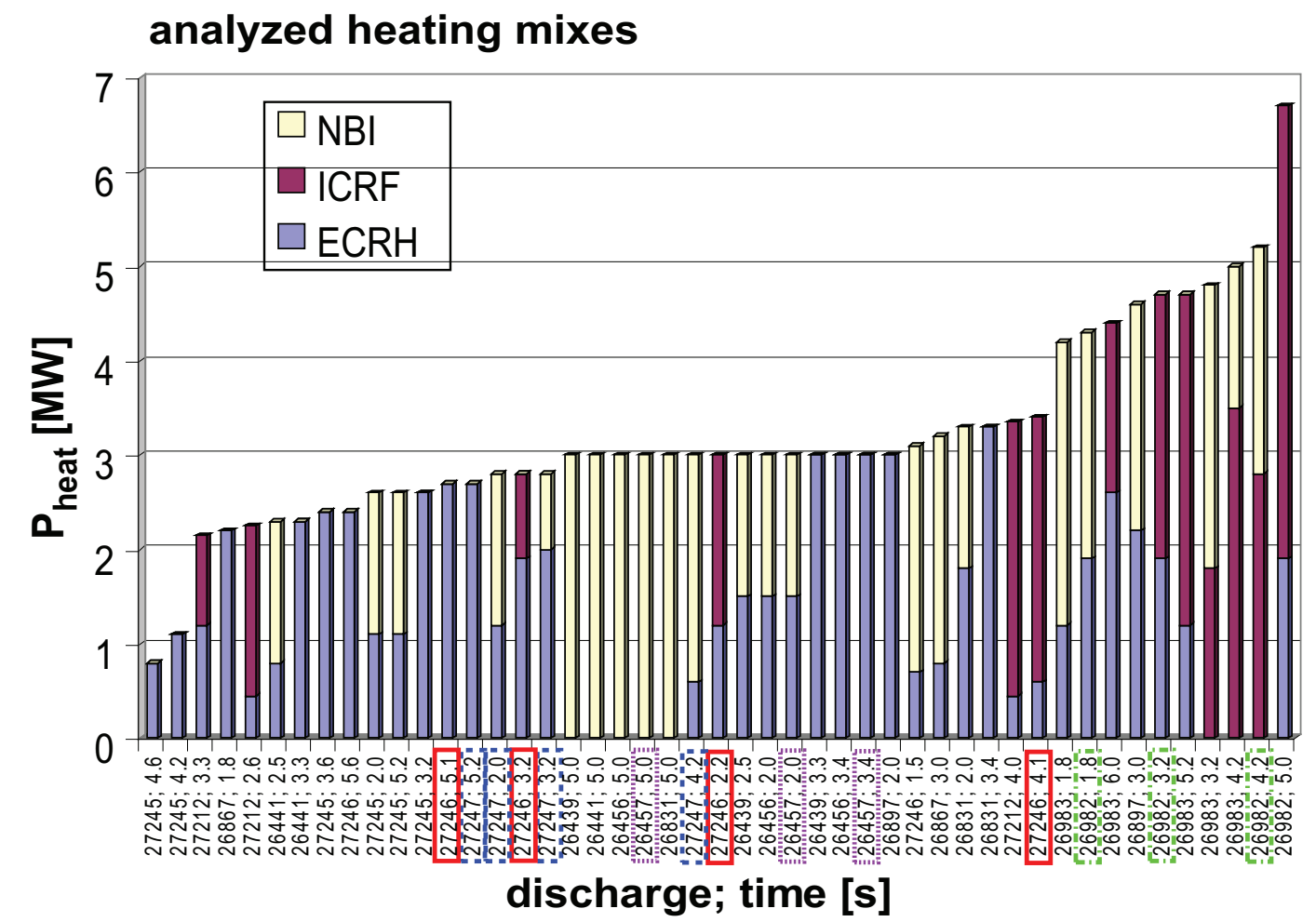

Figure 1. Overview of analysed cases comparing the heating mix of NBI, ICRF and ECRH. Highlighted with boxes are the cases covered in this paper. \# 27247 comparing ECRH vs. NBI (dashed blue boxes), \# 27246 comparing ECRH vs. ICRH (continuous red boxes), \# 26982 comparing ELM behaviour (dash dotted green boxes), \# 26457 used for GS2 calculations (dotted purple boxes).

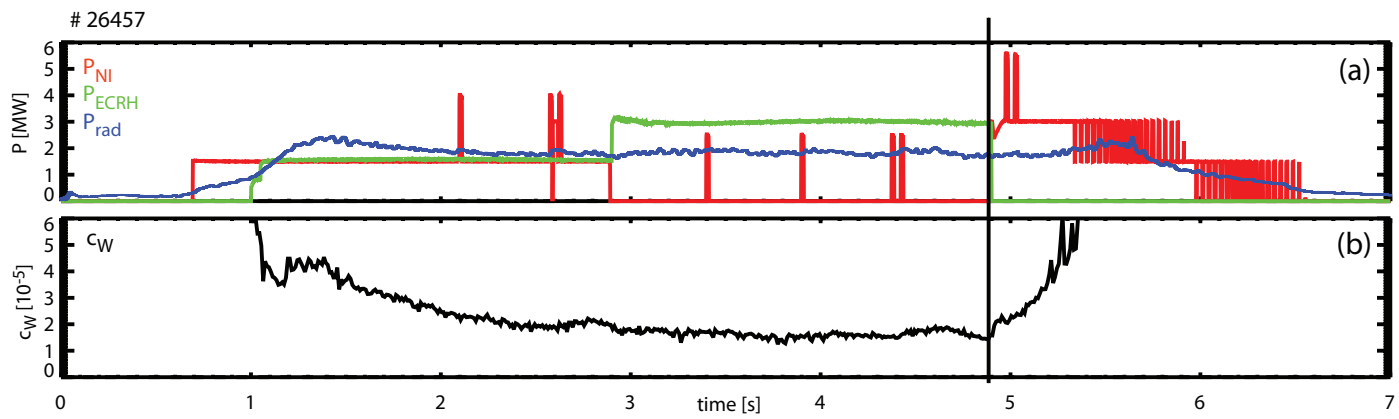

Figure 2. (a) NBI, central ECRH and radiated power; (b) Tungsten concentration

of $q_{95}=4.0$. The local central density lies in the range of $n_{e} \sim 9 \cdot 10^{19} \mathrm{~m}^{-3}$. Time traces of important plasma parameters from this discharge are shown in Figure 3. In the uppermost panel the total auxiliary heating power is shown in black. While it was kept constant at $3 \mathrm{MW}$, the fraction of ECRH and NBI power was varied in 4 steps during the discharge. Highlighted with coloured vertical bars are the four phases in which the ECRH constitutes half (green), three quarters (yellow), one quarter (red) and the total amount of the auxiliary heating power (blue). The total auxiliary heating power is around 2 times higher than the H-mode power 


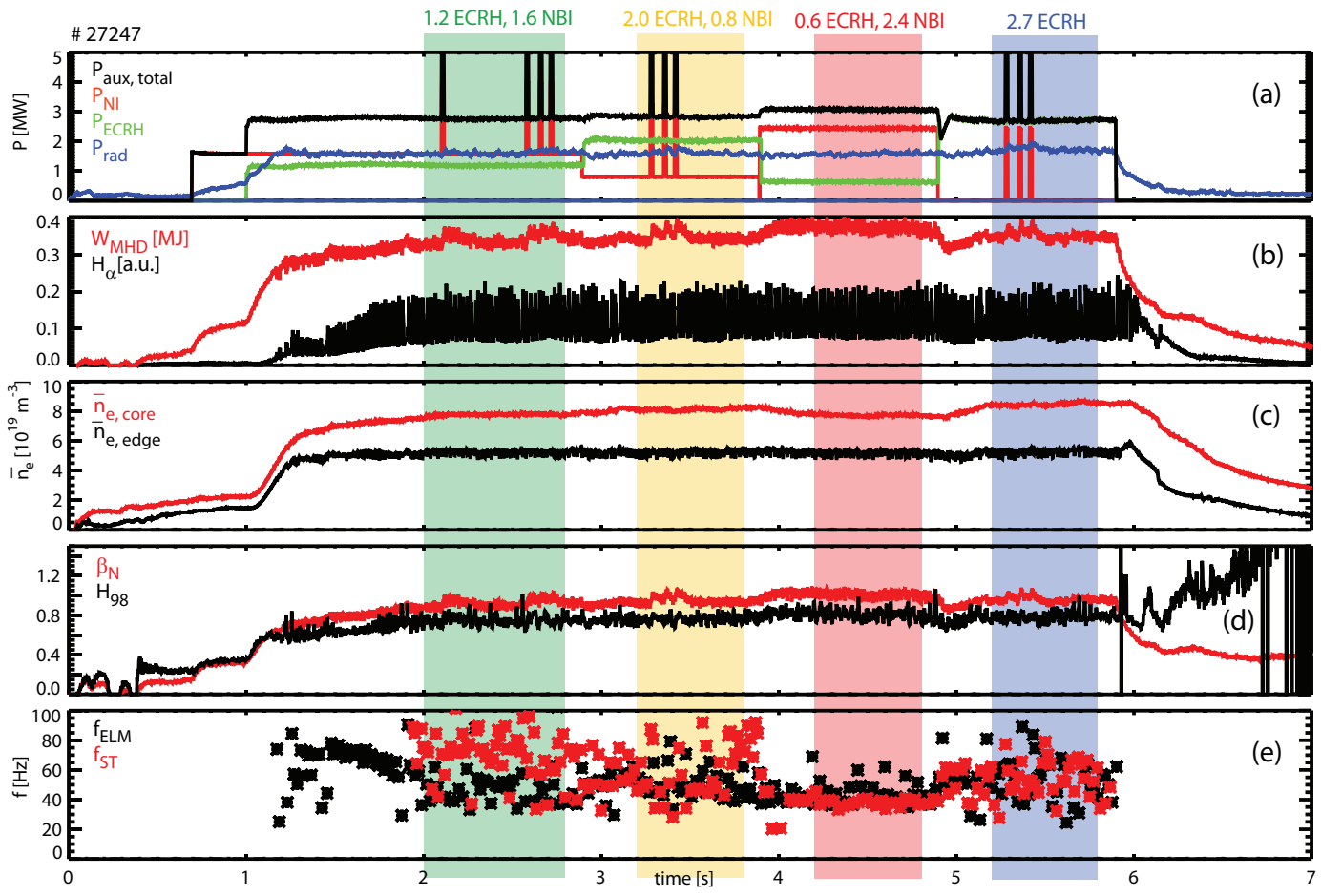

Figure 3. Global plasma parameters of discharge \# 27247: (a) total auxiliary, NBI, central ECRH and radiated power; (b) stored energy, $H_{\alpha}$ radiation; (c) line averaged density in the core and at the edge; (d) normalised beta, confinement factor; (e) ELM and sawtooth frequency

threshold at these densities [5]. The heating power of the NBI is shown in red. Several beam blips were introduced to measure the ion temperature $T_{i}$ and rotation $v_{t o r}$ in the core [6] and in the edge [7] with charge exchange recombination spectroscopy (CXRS) in the phases with reduced beam heating. These blips can be seen in Figures 3 and 5 as spikes in the time traces of the total and NBI heating power. Each group of three blips was synchronised with a scan of the low field side separatrix position $R_{\text {out }}$ in a way that the radial resolution of the measurement is maximised while keeping the disturbance of the plasma as small as possible. Neither the electron density (Figure 3 (c)) nor the electron temperature are affected by the short blips. The three frames of the CXRS measurement within one beam blip show some scatter, but no tendency towards higher $T_{i}$ values within one blip is evident. No beam blips are needed in the third phase of discharge \# 27247, since the beam at which the diagnostic is looking at is switched on the whole time for heating. The ECRH power is shown in green. In all analysed discharges its deposition location was in the very centre at $\rho_{\text {pol }}=0.1-0.2$. No toroidal angle was introduced in order to have no current drive by ECCD. The radiated power, measured by bolometry is shown in blue.

Despite significant changes in the heating mix, the global plasma parameters are almost constant over the entire discharge. The $H_{\alpha}$ signal (black, Figure 3 (b)) shows no significant change. Also, the stored energy $\left(W_{M H D} \sim 350 \mathrm{~kJ}\right.$, red, Figure 3 (b)), the normalised beta $\left(\beta_{N} \sim 0.9\right.$, red, Figure $\left.3(\mathrm{~d})\right)$, the confinement factor $\left(H_{98} \sim 0.8\right.$, black, Figure $\left.3(\mathrm{~d})\right)$ and the 
ELM frequency $\left(f_{E L M} \sim 50 \mathrm{~Hz}\right.$, black, Figure $\left.3(\mathrm{e})\right)$ are invariant. The sawtooth frequency (red, Figure 3 (e)) is in the same range, but shows a much larger scatter from around 40 to $100 \mathrm{~Hz}$. The volume averaged effective collisionality, $v_{\text {eff }}=0.1 \cdot R \cdot Z_{e f f} \cdot\left\langle n_{e}\right\rangle /\left\langle T_{e}\right\rangle^{2}$ [8], is around 2 for the entire discharge.

From the evolution of the electron temperature (not shown) a transition time between the different phases of around $50 \mathrm{~ms}$ can be seen. This is in the range of the energy confinement time. The line averaged density shows a slower transition behaviour and takes around $200 \mathrm{~ms}$ to reach a new steady state (Figure 3 (c)). After these transitions the plasma parameters are fairly constant except for two modulations: On one side the core temperature is modified by the sawtooth oscillations [9] while the variation in the density is negligible. On the other side both temperature and density at the edge are expelled by ELMs. The measurement of $T_{i}$ and $v_{\text {tor }}$ via beam blips could only be done for a few time points, which have an arbitrary phase to the sawtooth and ELM cycle. Therefore it is not possible to resolve these MHD instabilities and all kinetic profiles are averaged over the constant period for each heating phase. This average over roughly 0.6 seconds includes at least 20 sawtooth and ELM cycles and allows reliable kinetic profiles to be compiled, which can be compared to each other.

The resulting kinetic profiles can be seen in Figure 4. For each phase of the discharge with varying heating mix all available profile data was fitted with a splinefit to be able to compare them among each other. The top row shows the electron and ion temperature, the electron density and the toroidal plasma rotation for the entire plasma radius. The second row shows the same data, enlarged for the edge region while only the data between $5 \mathrm{~ms}$ after an ELM until $2 \mathrm{~ms}$ before the next ELM was considered. Note the different $\rho_{p o l}$ scale of Figure 4 (f). An additional phase featuring 3 MW of NBI only heating taken from discharge \# 26457 has been included in these figures to complete the power mix scan. The characteristics of this discharge are very similar to \# 27247 (same $I_{P}, B_{t}, n_{e}$, shape) and no operational deviations are known, which would explain the differences in the resulting profiles. So the comparability is given. Due to the increased electron heating the central electron temperature increases by $20 \%$ when only $600 \mathrm{~kW}$ of ECRH power is applied. Further increases of the electron heating fraction, however, have no measurable influence on the electron temperature profile (Figure 4 (a)). The ion temperature is reduced by $15 \%$ when applying at least $1.2 \mathrm{MW}$ of the total power by ECRH (almost 50\%). This effect also saturates and the ion temperature profiles stay constant even as the ECRH power fraction is increased to $100 \%$. The electron temperature inside the pedestal top gradually declines around $10 \%$ when going from NBI only to ECRH only heating (Figure $4(\mathrm{~d})$ ) which is only barely outside the scatter of the measured data at the edge. Outside of the pedestal top the identification of the electron temperature with the radiation temperature measured by the ECE is no longer justified due to the steep gradients and the low optical depth of the scrape off layer plasma. Therefore, no conclusion about the behaviour of the electron temperature in this region can be drawn. The variation of the ion temperature at the edge lies well within the scatter of the original data. 

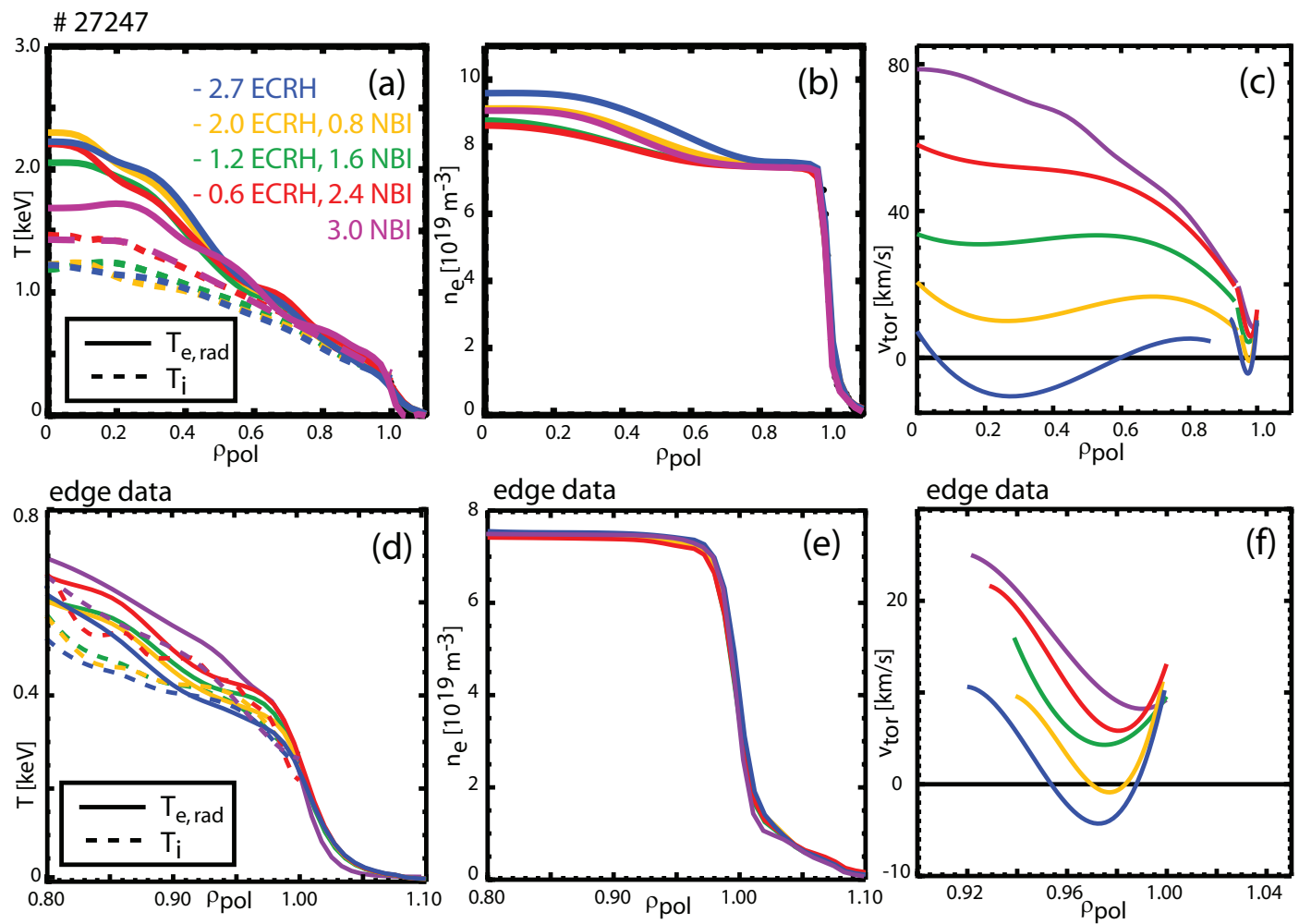

Figure 4. Averaged kinetic profiles of discharge \# 27247 during different heating phases: (a) electron (solid) and ion (dashed) temperature; (b) electron density; (c) toroidal rotation; Purple traces taken from discharge \# 26457, (d), (e), (f) edge data; note different $\rho_{\text {pol }}$ scale of (f)

The decreasing fraction of NBI heating results in a reduced torque input by the beams. This leads to the observed strong decrease of the toroidal rotation of the entire plasma (Figure 4(c)). The data from the core CXRS and from the edge CXRS system were fitted separately to account for the different radial resolution and scatter of the two diagnostics and to recover the localised structures at the edge. The rotation reaches even negative values in the core for the ECRH only case. The toroidal rotation $v_{t o r}$ at the edge shows the same behaviour (Figure 4 (f)). The local minimum of the toroidal rotation and the inversion of the rotation shear around $1 \mathrm{~cm}$ inside the separatrix is typically observed in H-mode plasmas at ASDEX upgrade and described in [10]. The poloidal rotation $v_{p o l}$ at the edge has small values and does not show any change with respect to the different heating mixes (not shown).

The density profiles consist of the data of the lithium beam and the DCN interferometer, which are combined within the Integrated Data Analysis using forward modelling on the raw data [11]. The Lithium beam sets the profile at the edge up to the pedestal top, while the inner part of the profile is a deconvolution of the $5 \mathrm{DCN}$ interferometer channels. The pedestal top density and the entire edge density profile are not at all affected by the change in heating mix (Figure 4 (e)). All 5 cases are plotted in this figure, but the difference between them is smaller than the line width, so that not all profiles can be seen in the figure. The central density exhibits an increased peaking with increasing fraction of ECRH power (Figure 4 (b))[12] which 
leads to an overall increased density. The peaking of the density profile with increasing ECRH power is typically observed at these intermediate to high densities in $\mathrm{H}$-mode operation which are dominated by ion temperature gradient turbulence (ITG [13][14]). This stands in contrast to the typical density pump out, which is observed at lower densities and in L-mode with increased ECRH due to an increased trapped electron mode (TEM [15][16]) activity.

The remaining rotation in the ECRH only case represents the intrinsic plasma rotation without external torque input which is governed by the residual stress [6][17].

For the impurity ions $v_{p o l}$ is the main contributor in the calculation of the radial electrical field in the edge $\left(E_{r}=\frac{1}{n_{i} Z_{i} e} \frac{\partial p_{i}}{\partial r}-v_{p o l, i} B_{t o r}+v_{t o r, i} B_{p o l}\right)$. Since its value is very small and no change with heating mix could be observed, an initial analysis of the data indicates that the radial electrical field does not change when changing the heating mix.

\section{Experimental observations of ECRH versus ICRF heated H-modes}

Changing the heating from NBI to ECRH does not only change the heating mix but also modifies the torque input and thus the rotation of the plasma to a great extent. This rotation, its shear and thus the $E \times B$ velocity shear are thought to be some of the key players in the reduction of turbulent transport. For that reason, discharges were conducted, in which ICRF and ECRH power were exchanged in the same way as the NBI and ECRH in the previous section. Also in this discharge, the ECRH was mainly deposited in the centre around $\rho_{p o l}=0.1-0.2$. However in the ECRH only phase the deposition locations of two of the six used gyrotrons are moved to mid radius $\left(\rho_{p o l}=0.5-0.6\right.$ ) due to a miss programmed control system. This change does not seem to have a noticeable influence on the global plasma parameters or kinetic profiles. The ICRF heating system was operated at a frequency of $36.5 \mathrm{MHz}$ for $\mathrm{H}$-minority heating with $5 \%$ of Hydrogen. This leads to a broad, centrally peaked heating profile. Like the NBI, roughly half of the ICRF heating energy is delivered to the electrons and half to the ions, but more peaked towards the centre. Time traces of key plasma parameters from discharge \# 27246 are shown in Figure 5. The depicted quantities are the same as in Figure 3. Figure 5 (a) contains the heating and radiated powers, the ICRF heating drawn in purple. As in discharge \# 27247 the different phases of one half, two third, one fourth and pure ECRH are highlighted with vertical bars. In this discharge the $H_{\alpha}$ signal (black, Figure 5 (b)) does not change with the different heating scenarios. The total stored energy ( $W_{M H D} \sim 350 \mathrm{~kJ}$, red, Figure $5(\mathrm{~b}))$, the normalised beta $\left(\beta_{N} \sim 1.0\right.$, red, Figure $\left.5(\mathrm{~d})\right)$ and the confinement factor $\left(H_{98} \sim 0.8\right.$, black, Figure $\left.5(\mathrm{~d})\right)$ remain constant throughout the discharge. The ELM frequency $\left(f_{E L M}=40-80 \mathrm{~Hz}\right.$, black, Figure $\left.5(\mathrm{e})\right)$ shows a large scatter but no fundamental change between the different heating phases. The sawtooth frequency (red, Figure 5 (e)) is in the range of $f_{S T}=50-100 \mathrm{~Hz}$ in phases with at least one third of ECRH heating. However in the case with the least ECRH power, it drops to $f_{S T} \sim 30 \mathrm{~Hz}$, discussed later. The effective collisionality $v_{e f f}$ is around 3 for the entire discharge. 

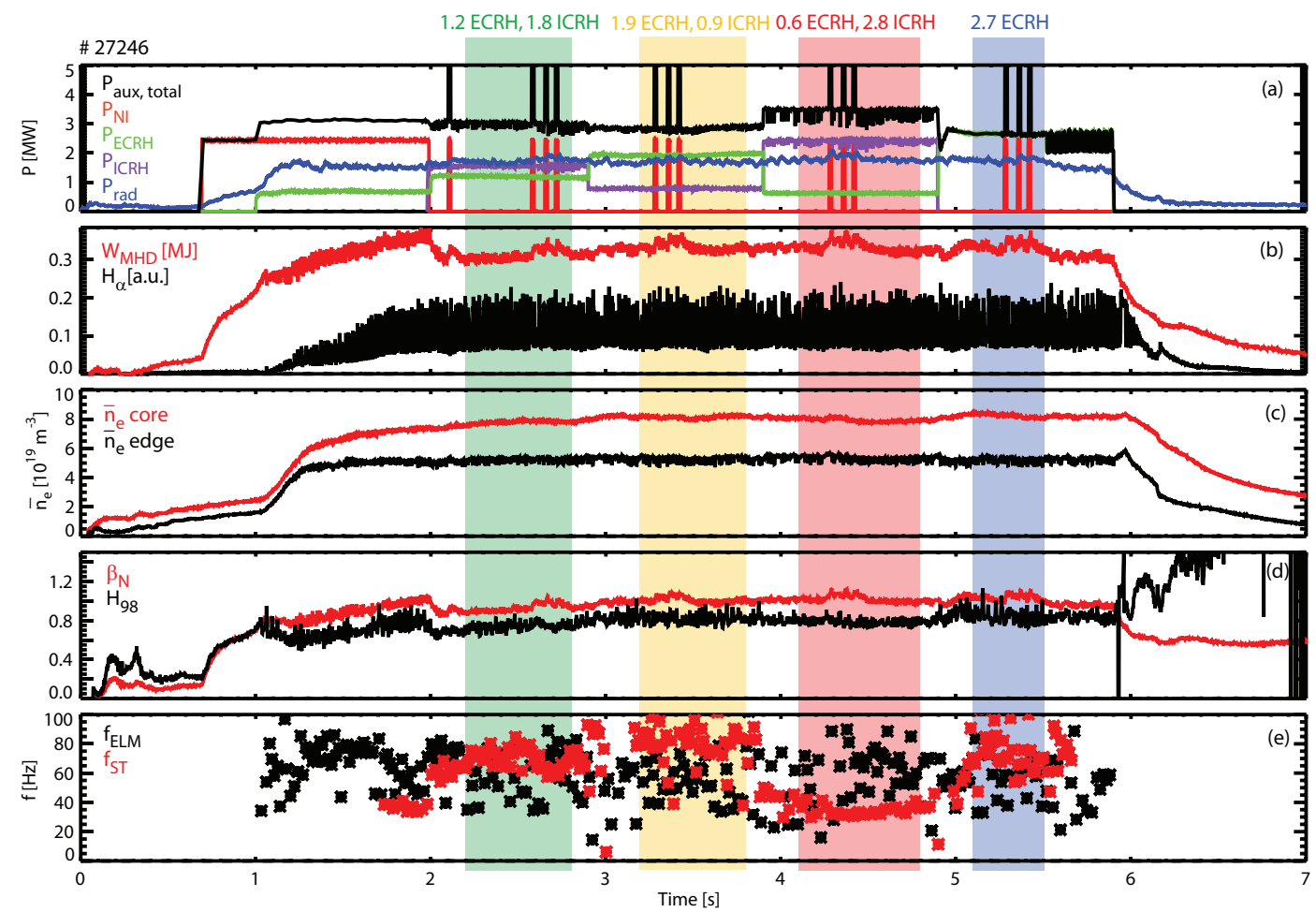

Figure 5. Global plasma parameters of discharge \# 27246: (a) total, NBI, central ECRH, ICRF heating and radiated power; (b) $H_{\alpha}$ radiation, stored energy; (c) line averaged density in the core and at the edge; (d) normalised beta, confinement factor; (e) ELM and sawtooth frequency

The profiles, each averaged over the constant phase of each heating mix, are shown in Figure 6. The electron temperature from the different heating phases is shown in Figure 6 (a) as solid lines. The high central temperature in the case with the minimum ECRH power occurs due to an extremely low sawtooth frequency of $30 \mathrm{~Hz}$. This allows the temperature to stay at the saturated high level for most of the period and explains the observation. In the other cases no change of the electron temperature with variation of the heating mix can be observed. The ion temperature also does not show a significant variation with heating mix. So far no discharges could be performed comparing an ICRF only heated phase to a phase with only low ECRH power. The largest change in the kinetic profiles in Section 2 was observed going from NBI only heating to a little fraction of ECRH. This comparison is still a pending issue.

Exchanging the ICRF by ECRH gradually increases the density peaking in the core very similar to the case in which the NBI was replaced by ECRH (Figure 6 (b)). The pedestal top value and the steep gradient region, however, do not change (Figure 6 (e)), as in discharge \# 27247. At the edge, neither the electron nor the ion temperature show changes of the profiles when changing the heating mix.

Since the torque input of the ICRF heating is very small, the toroidal rotation also stays below $10 \mathrm{~km} / \mathrm{s}$ and diminishes only slightly in the main plasma region with reduced ICRF heating 

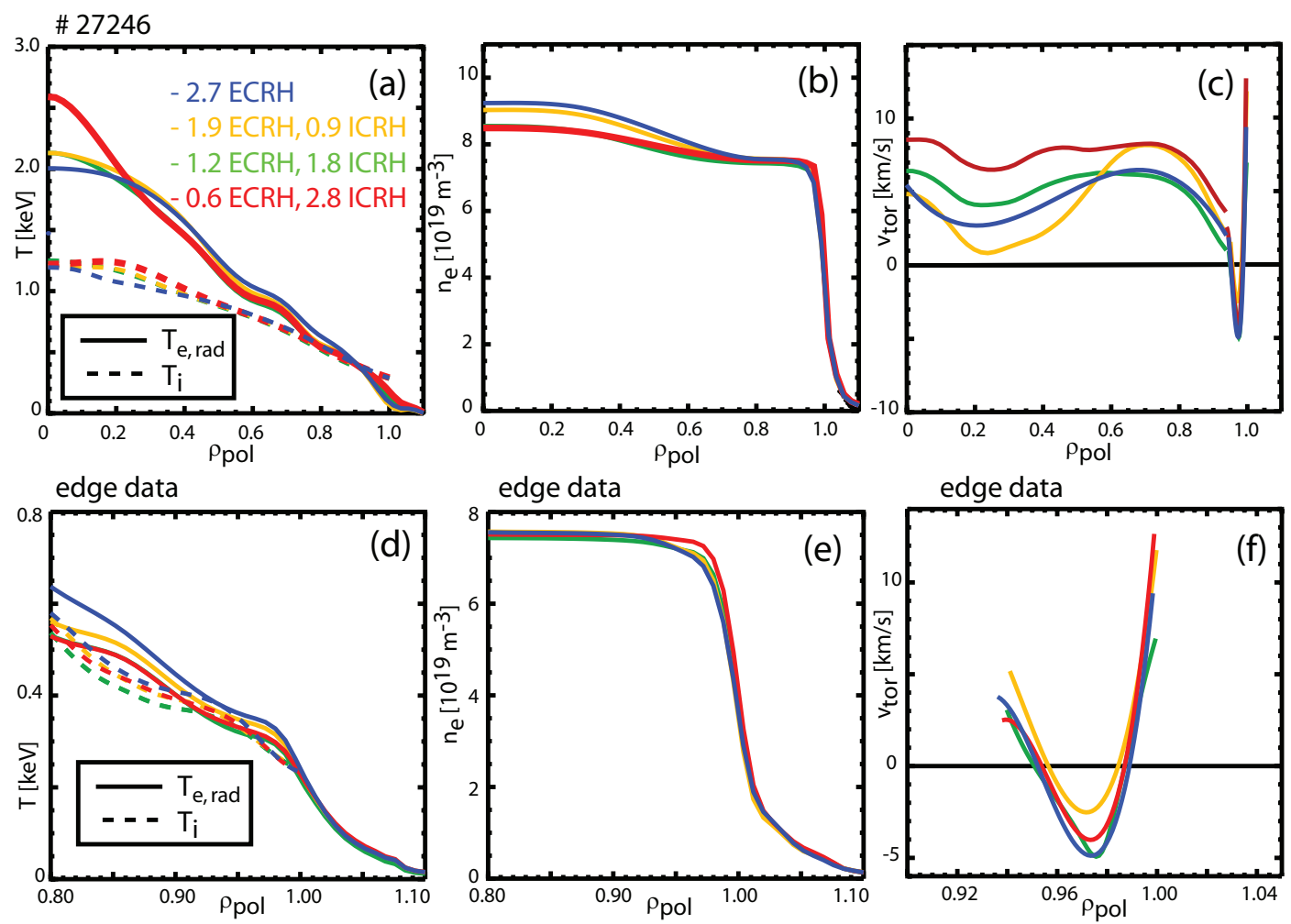

Figure 6. Averaged kinetic profiles of discharge \# 27246 during different heating phases: (a) electron (solid) and ion (dashed) temperature; (b) electron density; (c) toroidal rotation; (d), (e), (f) edge data. Note that Figure 6 (c) has a different scale in comparison to Figure 4 (c) by a factor of 4 and Figure 6 (f) has a different scale in comparison to Figure 4 (f) by a factor of 2.

fraction. Note the reduced scale of Figure 6 (c) in comparison to Figure 4 (c) by a factor of 4 and of Figure 6 (f) in comparison to Figure 4 (c) by a factor of 2.

These observations can be compared with those from Section 2, in which NBI power was replaced step-wise with ECRH in discharge \# 27247. The only change from the operational point of view between these two discharges is the replacement of the ICRF by NBI heating. Figure 7 shows the kinetic profiles from the case with half NBI plus half ECRH power (red) and the case with half ICRF plus half ECRH power (green). The most obvious change is the core and edge rotation of the plasma. Neither the global plasma parameters like stored energy or confinement nor the shape of the density and ion temperature profiles or their responses to ECRH show any significant variation outside the scatter of the original data. The pedestal top value of the electron temperature is slightly higher in the NBI case. The differences in the center of the radial profiles lie within the uncertainties of the original data and the deviation in the gradients outside the separatrix are due to the fact, that the plasma is getting optically thin in this outer region, so that the real plasma electron temperature is not equal to the radiation temperature anymore. Apparently the large change in rotation does influence the heat transport only a little at the edge of the plasma and has no detectable impact in the center and on the particle transport. 

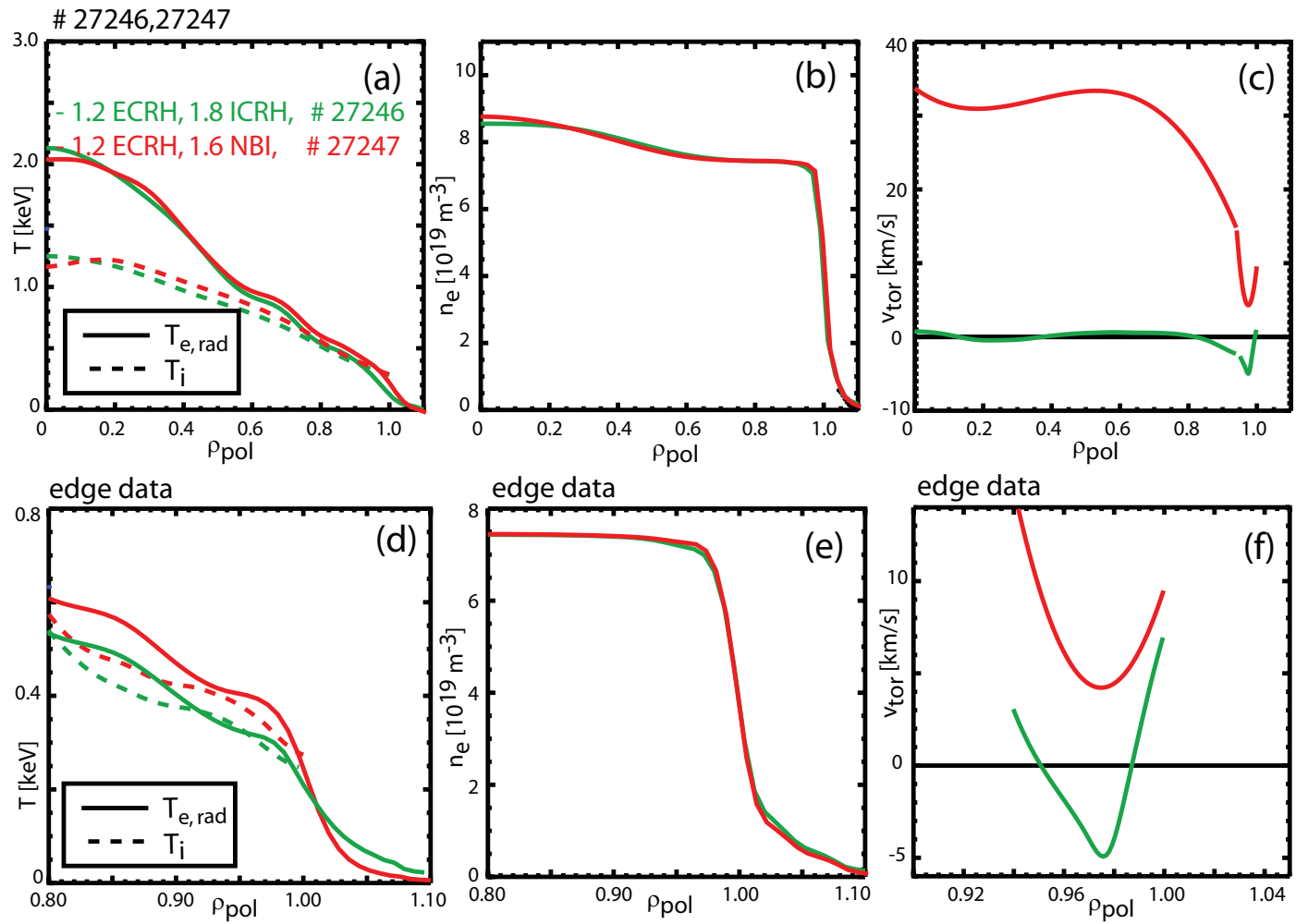

Figure 7. Averaged kinetic profiles from discharges \# 27246 and \# 27247 during phases with half ECRH plus half ICRF and half ECRH plus half NBI heating: (a) electron (solid) and ion (dashed) temperature; (b) electron density; (c) toroidal rotation; (d), (e), (f) edge data

\section{Experimental observations of ELM behaviour}

Variation of the heating mix does not have an apparent influence on the pedestal and on the steep gradient zone. However a change in the ELM behaviour was found in the course of the analysis. To analyze this behaviour a discharge very similar to the ones described above was performed with three phases, each combining two of the available heating methods: $2.4 \mathrm{MW}$ NBI plus 1.9 MW ECRH, 1.9 MW ECRH plus 2.8 MW ICRF and 2.4 MW of NBI plus 1.8 MW ICRF heating. This was necessary to rule out the change of plasma, vessel and divertor conditions between the different phases to a large extent. The described effects of the different heating mixes on the ELMs could be observed in all other analysed discharges, however not always as pronounced as presented here. Figure 8 shows the time traces of ELM behaviour relative to the ELM onset time for the three different heating mixes in discharge \# 26982. The first column shows data from a phase heated with about half NBI and half ECRH. The second column with about half ICRF and half ECRH and the third with about half NBI and half ICRF heating. In each case, the data from roughly 20 ELMs is over plotted. The first two rows show two channels of the AXUV diode bolometer looking from the top to the inner (a) and outer (b) divertor [18]. Figure 8 (c) depicts the scrape-off-layer current flowing to the inner divertor [19][20], Figure 8 (d) the deposited power on the inner divertor, as measured with thermography [21] and Figure 8 (e) the loss in stored energy normalised to the value of 

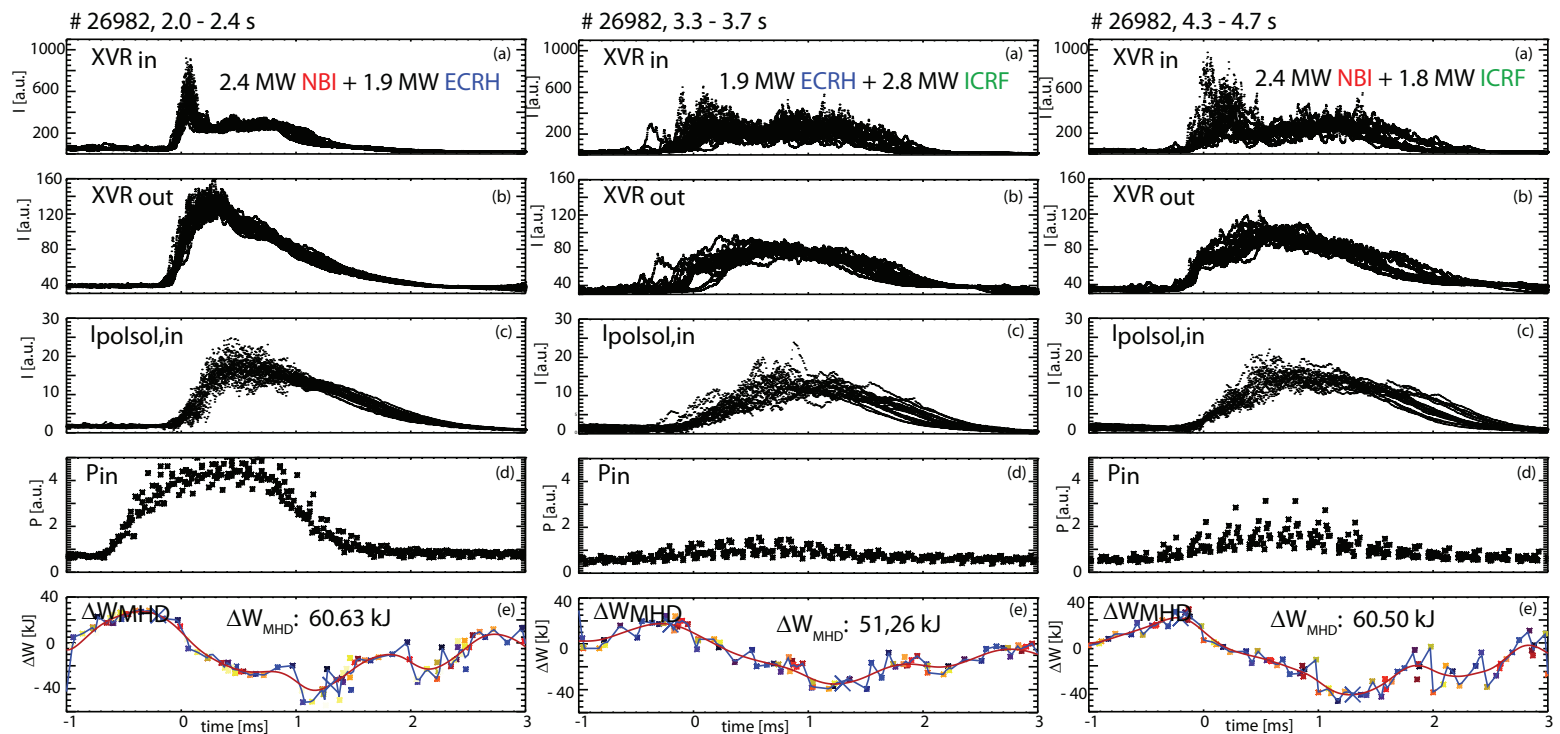

Figure 8. ELM onset synchronised time traces for discharge \# 26982: diode bolometer looking from the top to the (a) inner and (b) outer divertor; (c) scrape-off-layer currents to the inner divertor; (d) deposited power on the inner divertor, measured with the thermography; (e) loss in stored energy normalised to $W_{M H D}$ before the ELM onset

$W_{M H D}$ before the ELM.

The measured time traces of the different ELMs overlap very nicely showing very good reproducibility. On the other hand the data shows a strong variation between the different heating phases. A distinct feature of the NBI plus ECRH case is the strong peak at the beginning of the ELM visible in the radiation signal both in the inner and outer divertor. It is much flatter in the other two cases. The divertor current has a steeper rising edge, and a peak value which is 15 - $30 \%$ higher than in the NBI plus ICRF and NBI plus ICRF cases. The heat deposition on the divertor plates is strongly pronounced, while it is almost not measurable in the other two cases. Despite the flatter time traces in the ECRH plus ICRF and the NBI plus ICRF cases the loss in stored energy is very comparable between the different heating mixes.

The observed effect of the strong rise in the divertor measurements in the first case likely results from an interplay between NBI and ECRH. It is not only caused by the NBI heating, since it is a lot less pronounced in the NBI plus ICRF heating case.

\section{Heat transport analysis}

The discharge comparing NBI with ECRH was also analysed with respect to the heat transport with ASTRA [22]. The results of this interpretative heat transport analysis are pictured in Figure 9. The first row shows the radial profiles of the power deposited by the ECRH to the electrons (Figure 9 (a)), by the NBI to the electrons (Figure 9 (b)) and by the NBI to the ions (Figure 9 (c)). By integrating the two NBI deposition profiles over the plasma volume, 


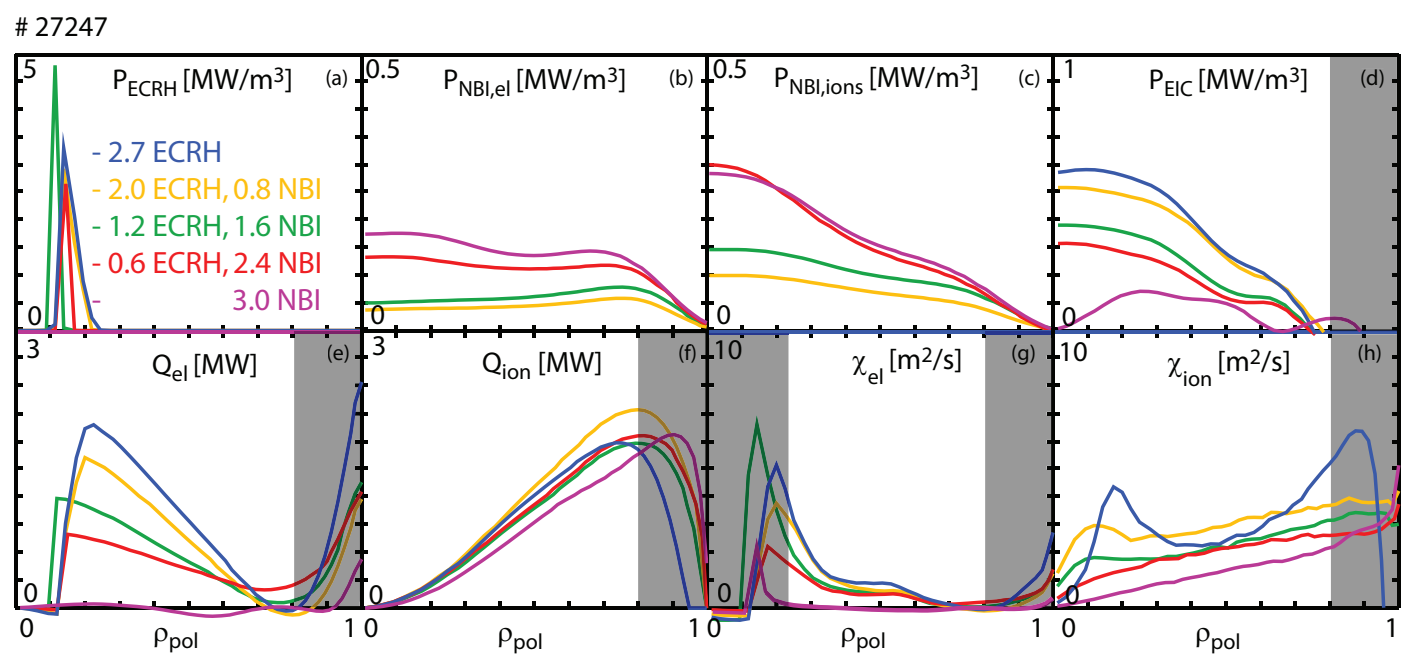

Figure 9. Heat transport profiles of discharge \# 27247 during different heating phases, (a) ECRH power density to electrons; (b/c) NBI power density to electrons/ions; (d) electron-ion heat exchange due to Coulomb collisions; surface integrated electron (e) and ion (f) heat flux; $(\mathrm{g})$ electron and $(\mathrm{h})$ ion heat diffusivities; Purple traces taken from discharge \# 26457

one can see that the heating power delivered to the electrons and to the ions is almost equal, but the radial distribution differs. The power transferred from electrons to ions by Coulomb collisions is depicted in Figure 9 (d). A very strong increase of this exchange term with increasing ECRH fraction is evident. This arises from an increased temperature difference and increasing density. Since $T_{e}$ and $T_{i}$ are very similar in the edge region, the exchange term is very sensitive to small variations in the temperatures, which cannot be resolved by the measurement. For that reason, the heat fluxes $Q$ and the heat diffusivities $\chi$ in the edge cannot be trusted (region marked with gray shaded vertical bars).

The second row shows the radial profiles of the electron and ion surface integrated heat fluxes $\left(Q_{e l}\right.$ Figure 9 (e) and $Q_{i o n}$ Figure 9 (f)). $Q_{e l}$ naturally shows a strong increase at the location of the ECRH deposition which increases with ECRH heating power due to a larger energy input. In the case of NBI only heating, the power going to electrons by NBI and ohmic heating cancels almost entirely with the radiated power and the power going to ions by Coulomb collisions over the whole radius. Towards the edge around $\rho_{\text {pol }} \sim 0.8$ the electron heat flux goes down to zero because all the energy is transferred to the ions. As a result, $Q_{i o n}$ shows a steady increase towards the edge. $Q_{i o n}$ has a tendency towards slightly higher values with increasing ECRH fraction over the whole plasma radius. The electron and ion heat diffusivities $\chi_{e l}$ and $\chi_{i o n}$ are shown in Figure $9(\mathrm{~g})$ and $(\mathrm{h})$. The grey shaded areas in the center of the plasma should not be considered since ASTRA has difficulties coping with the strong gradients near the very localised ECRH power deposition location and a limited radial resolution. The electron heat diffusivity is around zero in the NBI only case since the electron heat flux is negligible. In all other cases it has a value of around $1 \mathrm{~m}^{2} / \mathrm{s}$ at mid radius and does not show any change with the heating mix within the uncertainties of the evaluation. The ion 

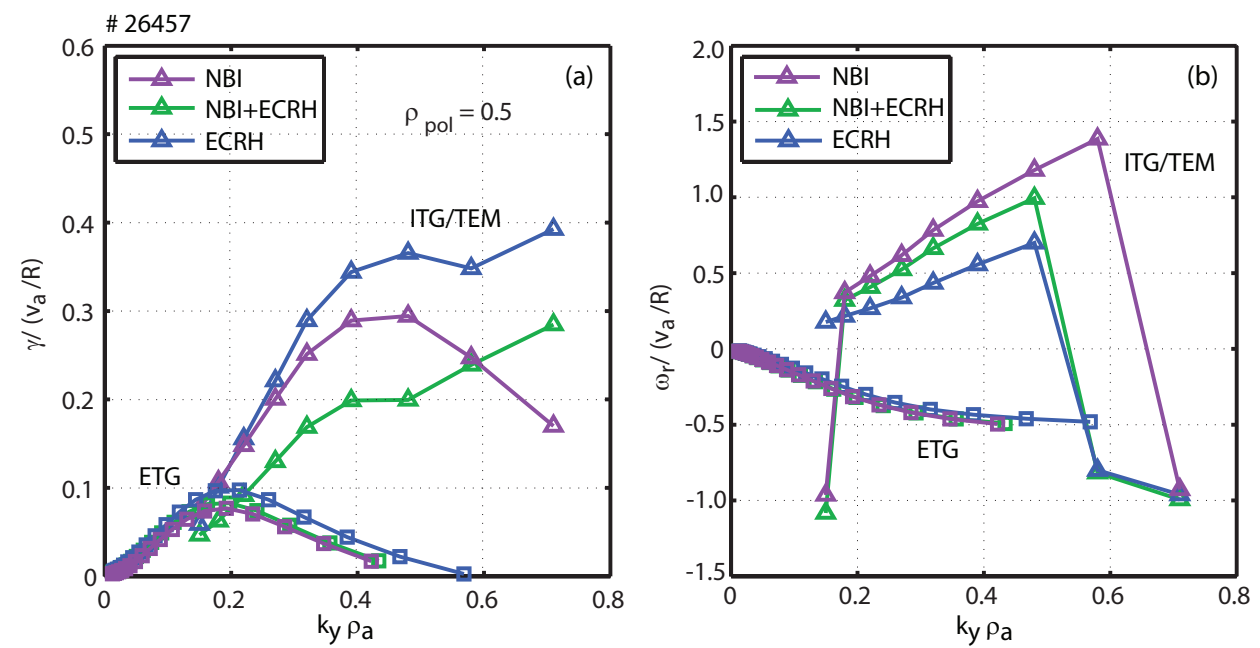

Figure 10. (a) growth rate of microturbulent instabilities; (b) frequency of microinstabilities. The index a in the labels denotes the species typical values to which the growth rates, frequencies and wave numbers of the instability are normalised. Ions for ITG and TEM mode and electrons for ETG mode

heat diffusivity shows a steady increase from around $1.5 \mathrm{~m}^{2} / \mathrm{s}$ in the NBI only case to $3 \mathrm{~m}^{2} / \mathrm{s}$ in the ECRH only case. This strong energy transfer from the electron to the ion channel is accountable for the only slight change of the kinetic profiles. This results in the invariance of the global plasma parameters and performance when going from NBI heating to pure ECRH.

In addition to this interpretative heat transport analysis, the nature of the underlying microturbulence responsible for the heat transport was also explored. On one hand in fusion plasmas the electron temperature gradient mode (ETG) [23] can be present, an electron gyroradius sized instability. On the other hand also ion gyroradius sized instabilities can occur which are the ion temperature gradient mode (ITG) and the trapped electron mode (TEM). The theoretical growth rates and the frequencies of these modes were calculated with the linear gyrokinetic code GS2 [24] for discharge \# 26457 at mid radius for the three heating phases NBI only, half NBI plus half ECRH and ECRH only. The growth rate and the frequency have been normalised to $v_{e} / R$ and wave numbers to $\rho_{e}$ for ETG modes, whereas to $v_{i} / R$ and $\rho_{i}$ for ITG modes. The growth rate is shown in Figure 10 (a) where the normalization leads to two peaks in the spectrum, one for the ETGs, one for the ITGs and TEMs. The growth rate of the latter exceeds the growth rate of the ETG at least by a factor of 3 for all cases, so it can be expected that the transport is dominated by ion orbit sized microinstabilities. In Figure 10 (b) the mode frequency is plotted, again showing two separate features for the ETGs and the ITGs/TEMs. The positive values of the ITG/TEM branches for all three heating mixes implies a rotation in the ion diamagnetic drift direction, which identifies the ITG as the dominant mechanism of radial heat transport. No difference in the fundamental nature of the turbulent transport could be observed, since the situation is the same for all three heating mixes. However a progressive reduction of the mode frequency going from NBI only heating to ECRH only is evident. This is a sign of increased influence of the TEM when going to higher electron heating fraction, 
but it does not take over the turbulent transport.

\section{Summary and outlook}

Experiments were carried out in which the mixture of electron and ion heating was varied from around $50 \%$ (NBI only) to $100 \%$ (ECRH only) of electron heating, while the total heating power was kept constant. The analysis of these experiments does not show any degradation in the basic plasma parameters and performance when replacing NBI and ICRF heating by ECRH. Furthermore almost no difference between NBI and ICRF heating could be observed. The application of low amounts of ECRH power leads to a slight increase in the electron temperature and a slight decrease in the ion temperature. Both effects saturate when ECRH power amounts to one third of the total applied power. With increasing fraction of ECRH a slight increase in density peaking and a severe drop in rotation can be observed. When going towards pure electron heating, the energy exchange term by Coulomb collisions is getting larger, so that the electron heat flux is approaching zero towards the edge of the plasma. On the other hand the heat flux in the ion channel is independent on the heating method and is increasing towards the edge. The nature of the underlying microinstability (ITG) does not change in this high collisionality domain when changing the heating mix. The ELM onset is much more pronounced in the cases of NBI and ECRH plus NBI heating compared to cases of ECRH only and mixed cases with ICRF heating. These first experiments on directly comparing ECRH with NBI and ICRF heating in H-modes show that the application of wave heating does not come with a degradation of plasma performance in the analysed parameter space. To widen this parameter space and to clarify the open questions further investigations will be carried out in the next campaign at ASDEX Upgrade.

\section{Acknowledgments}

The authors would like to thank Alberto Loarte for the stimulating discussions.

Partially funded by EFDA Grant WP10-TRA-02-01.

\section{References}

[1] D. Wagner, et al. Recent Upgrades and Extensions of the ASDEX Upgrade ECRH System. Journal of Infrared, Millimeter and Terahertz Waves, 32:274-282, 2011.

[2] Vl. Bobkov, et al. ICRF antenna-plasma interactions and its influence on W sputtering in ASDEX Upgrade. Journal of Nuclear Materials, 415(1, Supplement):S1005-S1008, 2011.

[3] H. Zohm. Edge Localized Modes (ELMs). Plasma Physics and Controlled Fusion, 38:105-128, 1996.

[4] R Neu, et al. Impurity behaviour in the ASDEX Upgrade divertor tokamak with large area tungsten walls. Plasma Physics and Controlled Fusion, 44(6):811, 2002.

[5] F. Ryter, et al. H-mode threshold and confinement in helium and deuterium in ASDEX Upgrade. Nuclear Fusion, 49(6):062003, 2009.

[6] R M McDermott, et al. Core momentum and particle transport studies in the ASDEX Upgrade tokamak. Plasma Physics and Controlled Fusion, 53(12):124013, 2011. 
[7] E. Viezzer, et al. High-resolution charge exchange measurements at ASDEX Upgrade. Submitted to Rev. Sci. Instrum.

[8] C. Angioni, et al. Scaling of density peaking in H-mode plasmas based on a combined database of AUG and JET observations. Nuclear Fusion, 47(9):1326, 2007.

[9] V. Igochine, et al. Comparison of different sawtooth crash models for transport analysis. 38th EPS Conf. on Plasma Physics. Contributed Papers, 35 G:P2.079, 2011.

[10] T Pütterich, et al. Evidence for Strong Inversed Shear of Toroidal Rotation at the Edge-Transport Barrier in the ASDEX Upgrade. Phys. Rev. Lett., 102:025001, Jan 2009.

[11] R. Fischer, et al. Multiple diagnostic data analysis of density and temperature profiles in ASDEX Upgrade. 36th EPS Conf. on Plasma Physics. Contributed Papers, 33 E:P1.159, 2009.

[12] C. Angioni, et al. Density response to central electron heating: theoretical investigations and experimental observations in ASDEX Upgrade. Nuclear Fusion, 44(8):827, 2004.

[13] B. Coppi and F. Pegoraro. Theory of the ubiquitous mode. Nuclear Fusion, 17(5):969, 1977.

[14] F. Romanelli. Ion temperature-gradient-driven modes and anomalous ion transport in tokamaks. Phys. Fluids, 1:1018 ff, 1989.

[15] J. Weiland, A.B. Jarmén, and H. Nordman. Diffusive particle and heat pinch effects in toroidal plasmas. Nuclear Fusion, 29(10):1810, 1989.

[16] B. Coppi and G. Rewoldt. New Trapped-Electron Instability. Phys. Rev. Lett., 33:1329-1332, Nov 1974.

[17] C. Angioni, et al. Intrinsic Toroidal Rotation, Density Peaking, and Turbulence Regimes in the Core of Tokamak Plasmas. Phys. Rev. Lett., 107:215003, Nov 2011.

[18] B. Reiter, et al. Application of AXUV Diodes for broad-band plasma radiation studies in ASDEX Upgrade. 36th EPS Conf. on Plasma Physics. Contributed Papers, 33 E:P1.161, 2009.

[19] A. Kallenbach, et al. Electric currents in the scrape-off layer in ASDEX Upgrade. Journal of Nuclear Materials, 290-293(0):639-643, 2001.

[20] G. Pautasso, et al. Evolution of the halo current in ASDEX Upgrade disruptions. 37th EPS Conf. on Plasma Physics. Contributed Papers, 33 E:P2.152, 2010.

[21] A. Herrmann, et al. Energy flux to the ASDEX-Upgrade diverter plates determined by thermography and calorimetry. Plasma Physics and Controlled Fusion, 37(1):17, 1995.

[22] G. Pereverzev and P.N. Yushmanov. ASTRA Automated System for TRansport Analysis in a Tokamak. Technical Report IPP 5/98, IPP, Garching, Germany, 2002. IPP report.

[23] F. Jenko, W. Dorland, and G.W. Hammett. Critical gradient formula for toroidal electron temperature gradient modes. Phys. Plasmas, 8:4096 ff, 2001.

[24] W. Dorland, et al. Electron Temperature Gradient Turbulence. Phys. Rev. Lett., 85:5579-5582, Dec 2000. 